\title{
Seven
}

\section{BIG ACCIDENT QUANTUM COSMOLOGY}

Big Accident Cosmologists grant the objective existence of space/time/mass/ energy; but, they contend, it all adds up to nothing. All opposing forces within our closed universe balance out perfectly; and the net result is zero. If a perfectly balanced universe is closed, the net energy in that universe is nil. As Alan Guth puts it, "The Universe is the ultimate free lunch." According to the "free lunch" interpretation of quantum physics, our universe is a delicate but accidental balance of negative and positive forces that sums up to nothing. This perfect balance is called "perfect symmetry."

\section{The Universe as a Big Accident}

If symmetry is perfect on a cosmic scale, the total amount of energy in the universe is actually zero. Does this mean that nothing caused the universe? If our universe is an absolute zero, absolutely nothing seems required to cause it! Is our universe such an ultimate absolute accident? Is it a nothing that is caused by nothing for no reason or purpose at all? Extreme Big Accident Cosmology answers affirmatively. This cosmology is advocated by Quantum Cosmologists like Edward P. Tryon, ${ }^{2}$ Peter Atkins, ${ }^{3}$ A. Vilenkin, ${ }^{4}$ Victor J. Stenger, ${ }^{5}$ Quentin Smith, ${ }^{6}$ and a few others ${ }^{7}$ for whom the origin of our universe was indeed a stupendous accident, having no cause whatsoever.

Cosmologists who take this final step are not Antecedent Universe Cosmologists because they acknowledge no antecedents at all, no oscillating predecessors, not even Mother Spacetime and the unstable energy situation of the physical vacuum, not even really empty space itself. They view our world as an absolute accident, requiring neither necessary nor sufficient conditions for its existence. They believe that our universe was created not only from nothing but by nothing. As Edward P. Tryon put it, "Our universe is simply one of those things which happen from time to time."' Tryon was only a forerunner of Big Accident Cosmology who suggested that our universe may be produced by a quantum fluctuation of "the vacuum of some larger space in which our Universe is imbedded,"' so he actually presupposed the pre-existence of something after all. Renunciation of all antecedent conditions by real Accidentalists like Peter Atkins and Victor J. Stenger is much more complete. As Atkins expressed it,

In the beginning was nothing. Absolute void, not merely empty space. There was no space; nor was there time, for this was before time. The Universe was without form and void.

By chance was a fluctuation, and a set of points, emerging from nothing and taking their existence from the pattern they formed, defined a time. 
The chance formation of a pattern resulted in the emergence of time from coalesced opposites, its emergence from nothing. From absolutely nothing, absolutely without intervention, there came into being rudimentary existence. ${ }^{10}$

\section{And as Stenger put it,}

I picture the origin of the universe as follows: in the beginning there was a void more empty than a perfect vacuum, empty not only of particles and fields but of space and time as well. It had perfect symmetry and zero energy. It was as much nothing as nothing can be. A fluctuation in that void then occurred, generating our universe and perhaps countless others very different from ours. ${ }^{11}$

Quentin Smith, another Big Accident Cosmologist, rejects Tryon's "vacuum fluctuation in empty space" account of the origin of the universe,,$^{12}$ along with the principle of universal causation. He affirms that "The most reasonable belief is that we came from nothing, by nothing, and for nothing." 13 That is Big Accident Cosmology in a nutshell!

Extreme quantum Big Accident Cosmology proposes that the creation of our universe is the ultimate chance happening, a totally spontaneous quantum fluctuation in, from, of, and by pure nothingness. How plausible is the view that nothing caused the Big Bang?

\section{Critique of Big Accident Quantum Cosmology}

The fundamental affirmations and presuppositions of Big Accident quantum cosmology are empirically vacuous and should be dismissed as utterly meaningless by empiricist standards. "Absolutely nothing caused the Big Bang" presupposes that "Absolutely nothing once existed," but no conceivable experience could ever directly verify this affirmation. Any confirming or disconfirming experience would exist and would thus falsify the claim. No examples of absolute non-existence, or of causation by non-existence, could ever be given directly in any conceivable experience. Also, we cannot reason inductively about such things since we have no instances with which to start.

Aside from being utterly unintelligible experientially and inductively, Big Accident quantum cosmology is troubled by three extreme affirmations that make it utterly implausible as an explanatory hypothesis. Big Accident theorists are committed to the preposterous claims that: $A$. The universe exists in such perfect symmetry that its net energy equals zero. B. Natural quantum laws exist and function in a state of absolute nothingness. C. Causality must be totally abandoned at the point of ultimate origins. But why are these claims so preposterous? 


\section{A. Perfect Symmetry and Zero Energy}

Our universe was created out of nothing by nothing, for nothing, and is nothing, according to extreme Big Accident quantum cosmologists. All the forces operating in the universe exist in such perfect balance or symmetry that the net energy of the universe is absolutely zero. Supposedly, the kinetic energy of the initial explosion is perfectly balanced by the counter-tug of gravity; positive and negative electrical charges of material particles all ultimately cancel one another out, as do matter and antimatter. Everything in the universe is so arranged that absolutely everything adds up to absolutely nothing.

Victor J. Stenger explicitly links zero energy with creation by nothing, arguing that "Since the universe has zero total energy, no energy was required in its production."14 This extreme "caused by nothing" hypothesis would be false, however, if the universe contains the slightest bit of positive energy, if symmetry is in any way imperfect. Does the empirical evidence support perfect symmetry? The average density of all positive and negative electrical charges may be zero, but assuredly we have not totaled them all. Even so, at least three important facts about our world count heavily against the theory that all manifestations of energy are so equally balanced that they sum up to zero: $i$. We live in an open universe; ii. Matter decisively triumphs over antimatter; and iii. The kinetic energy of the initial bang is more powerful than gravity.

\section{i. We Live in an Open Universe}

First, only a closed universe can have zero energy, but overwhelming evidence, given in Chapter Three, indicates that our universe is open. Andrei Linde refers to "The well-known fact that the total energy of a closed Universe is zero, being a sum of the positive energy of matter and the negative energy of the scale factor $a . " 15$ But Linde's "well-known fact" is not really a fact about our universe. Free-lunchers do a lot of wishful thinking!

At this stage in the development of science, the most reasonable view is that our universe is open. Entities involved in the Hubble expansion have enough escape velocity to keep expanding forever; the Hubble rate of cosmic expansion is increasing, not decreasing; and gravitational and kinetic energy are decisively out of balance. After all identifiable dark matter is counted, seventy to ninety percent of the mass/energy required to close the universe is missing. Recall earlier discussions of the claim that sufficient mass exists to counterbalance the expansion of the universe. The missing mass just isn't there.

Many prominent scientific cosmologists believe that the universe is open and will expand forever, precisely because not enough matter or mass exists to close it, balance it, or even slow it down. ${ }^{16}$ Some cold dark matter exists; but prospects are dim that sufficient missing mass will ever be located to close down the expansion of the universe. ${ }^{17}$ This is especially obvious now that we 
know that the rate of Hubble expansion is increasing, not decreasing. Oddly, in his most recent book, Victor J. Stenger insists that we live in an open universe, one lacking enough mass to close it; ${ }^{18}$ but he inconsistently affirms that enough invisible dark matter exists to balance exactly the kinetic and rest energies of the mass of the universe. ${ }^{19}$ Stenger concedes, significantly, that what he means by "zero energy" actually contains a small positive amount of energy $!^{20}$ So, where did that tiny bit come from? Stenger offers no answer.

If enough mass/energy exists to register at .99999 instead of a measly .1 to 3 of Omega at 1 , the universe would still be open. Exactly at Omega, expansion would proceed very slowly forever; but the universe would never contract; a spent world would endure forever. Omega plus an additional scrap of mass/ energy is required for reversal. In the extremely unlikely event that our measurements might some day approximate these near-closure figures, margins of error would always prevent us from knowing for sure that the universe is closed.

Without sufficient mass/energy, gravity loses; kinetic energy wins; and the average net results are greater than zero. Victor J. Stenger claimed in his 1988 book that the universe is balanced between open and closed, ${ }^{21}$ yet, an impressive seventy to ninety percent of the evidence cannot be found. Stenger concedes this in his 1995 book where he announces that the universe is open, ${ }^{22}$ but he does not acknowledge that only a closed universe could be a Big Accident. Believing in a closed universe, or even a delicately balanced one, is too much like believing in Santa Claus and Ptolemaic epicycles. Abdus Salam concedes that "At the present time, measurements do not appear to sustain" the claim that "the mass of the universe adds up to zero;" and without this, "We shall discard the whole notion of the universe arising as a quantum fluctuation." ${ }^{23}$ Yes indeed!

\section{ii. Matter Prevails over Antimatter}

Second, our material universe exists precisely because matter and antimatter do not exist in perfect symmetry. We live in a material world because matter triumphed over antimatter near the very beginning, or because our universe was created from the outset with a massive imbalance of matter over antimatter. Stenger defends a hidden symmetry of matter and antimatter, despite the complete absence of evidence for it and substantial evidence against it. His only evidence is the non-empirical Principle of Plenitude. All possible states are actual, he insists. Symmetry is broken and matter prevails in our domain, he concedes, but in other domains (other universes, presumably composed of antimatter) in infinite Superspacetime, things balance out. ${ }^{24}$ But how does he know that? We have no empirical access to any Superspacetime containing antimatter universes, much less an infinitude of it! And Superspacetime, if it exists, is not sheer nothingness.

We do not know that any, much less that all, possibilities are actualized in other domains or universes somewhere in Superspacetime. This a priori meta- 
physics cannot be verified or falsified. Empirically, the claim is utterly meaningless, permanently without experiential content for us. An implausible hypothesis-creation of, by, out of, and for absolutely nothing-cannot be defended successfully by appeal to theories that are utterly senseless.

In this world, the only one that we know to exist, mass/energy is definitely not zero. As A. Zee wrote in 1986,

Ever since Dirac's ideas about antimatter were experimentally confirmed, people have speculated that the Universe has an equal amount of matter and antimatter, segregated into domains. More precisely, it was asserted that all conserved quantum numbers of the Universe should be zero. In particular, since electric charge $Q$ is known to be zero to a high degree of accuracy, it seems "aesthetically appealing" that baryon number B and lepton number L should also be zero. Unfortunately, the weight of the observational evidence is against this supposition. ${ }^{25}$

After thoroughly reviewing the empirical evidence, Gary Steigman concluded as early as 1979 that "The Universe is not symmetric and contains little, if any, antimatter." ${ }^{26}$ In 1991, John D. Barrow concurred:

Although particle accelerators produce matter and antimatter in equal abundances quite routinely and there is a democratic relationship between the two, we see no antiplanets, no antistars, no antigalaxies, and there is no evidence of any antimatter in the cosmic rays that come from outside our solar system. Nor do we see any evidence of the wholesale annihilation of matter and antimatter, which would erupt anywhere in the Universe, where the two came into contact. Thus, for some mysterious reason, there exists a form of cosmic favoritism. ${ }^{27}$

In 1993, after describing his research team's extensive probing during the 1970s for antimatter particles with sensitive instruments carried by high altitude balloons, George Smoot wrote that "During all the years, we found not a single convincing sign of cosmic antimatter. ${ }^{28}$

Perhaps, however, a perfect symmetry of matter and antimatter existed "in the beginning," even though little or no antimatter exists today. As Heinz R. Pagels suggests, "The present matter-antimatter asymmetry of the universe does not reflect the original state of the primal fireball, which could have perfect symmetry."'29 Even so, free-lunchers must explain how the original perfect symmetry of matter was broken and overcome, how enough matter to comprise our universe survived an initial perfect balance of matter and antimatter, why so little antimatter survived, and how this all adds up to nothing.

A widely accepted explanation is that a surplus of matter over antimatter resulted because the earliest physical particles decayed at an irregular pace, as 
permitted by the weak force. ${ }^{30}$ This answer is very problematic. Why did the weak force permit asymmetrical decay into matter instead of antimatter? Why didn't the weak force exemplify perfect symmetry? Emerging from perfect symmetry, why were these primitive particles asymmetrically inclined? Why weren't they all annihilated by their own perfectly symmetrical antiparticles?

This widely accepted explanation pushes the asymmetry back one step but does not account for it. No one has ever observed the primitive particles that supposedly decayed into a surplus of matter; they are purely hypothetical explanatory constructs with no empirical status whatsoever. Most seriously, if symmetry was so perfect originally, all of the original primitive particles should have been annihilated by their own primitive anti-particles. The conventional explanation of why asymmetry exists in a perfectly symmetrical universe is unverified, incoherent, and unintelligible.

In 1998, A. G. Cohen, A. De RúJula, and S. L. Glashow indicated that primordial matter/antimatter symmetry would elevate the gamma ray background and distort the cosmic microwave background far above observable quantities. They argue that although small pockets of antimatter might exist here and there, empirical evidence excludes a patchwork universe composed of widely separated regions of matter and antimatter. After reviewing the evidence, they conclude that " $\mathrm{A}$ matter-antimatter symmetric universe is empirically excluded." ${ }^{31}$

Even if equal quantities of matter and antimatter existed originally, this would still not prove zero net energy for the universe as a whole. When material and antimaterial particles collide and explode, they do not leave behind zero energy or absolute nothingness, as the "free lunch" Big Accident theory predicts and requires. Instead, they leave a residue of gamma radiation, which is a definite and positive form of mass/energy. ${ }^{32}$ In fact, residues of primordial gamma radiation may now have been found, ${ }^{33}$ but the early mutual extinction of matter and antimatter did not result in zero mass/energy. Clearly, the primordial annihilation was asymmetrical, a fact that perfect symmetry cannot explain.

\section{iii. The Bang Overpowers Gravity}

Before concluding that the universe is really not a free lunch after all, let us consider another argument for this conclusion. If matter/antimatter symmetry does not add up to zero, perhaps matter-gravity symmetry does. In explaining Stephen Hawking's commitment to the zero-energy free lunch theory, Michael White and John Gribbin declare that "If all the matter in the Universe could be collected together at a single point, its negative gravitational energy $\left(-m c^{2}\right)$ would exactly cancel out all the positive mass energy $\left(+m c^{2}\right)$ of all the matter." ${ }^{34}$ This argument for a zero energy universe completely ignores the stupendous kinetic energy of the Big Bang itself, against which gravity is fighting a losing battle. It was written before we discovered that the rate of 
Hubble expansion is increasing. It assumes incorrectly, as Hawking does (at times), that the universe contains enough mass/energy to close it.

Surprisingly, Hawking himself says in his 1988 A Brief History of Time that "The present evidence suggests that the universe will probably expand forever", ${ }^{35}$ but his no boundary model of the universe as a globe that begins at the North Pole, expands to the Equator, then shrinks to the South Pole, implies that the universe will not expand forever because an open universe that expands forever would have no South Pole. Which is the real Hawking?

In Stephen Hawking's A Brief History of Time: A Reader's Companion, published in 1992, Hawking says, "I predict that the universe in time will come to an end at the big crunch." ${ }^{36}$ In his 1993 book, Black Holes and Baby Universes and Other Essays, Hawking defends both the openness and the closedness of the universe and concludes that he is hedging his bets "by predicting both ways. ${ }^{" 37} \mathrm{He}$ concedes that observation discloses only ten percent of the mass required for closing the universe.$^{38} \mathrm{His}$ argument for a closed universe is purely conjectural, theoretical, and a priori.

Peter Coles and George Ellis wrote in 1994 that "The primary reasons for the widespread belief in a critical density of matter are theoretical." They emphasize that

this is indeed an experimental question, where theory-no matter how dear it may be to us-will eventually have to bow to the experimental evidence. It may be that the theoretical prejudice in favor of the high-density models will one day be confirmed; if so that will be a great triumph for theory. However, at present the weight of evidence if anything favors a lowdensity universe. ${ }^{39}$

After examining both theoretical and empirical evidence for a closed universe, Coles and Ellis affirm that "The amount of dark matter for which there is compelling direct evidence is a long way short of closing the Universe, ${ }^{140}$ and that "No strongly convincing case can be made for a critical-density Universe, and on the balance of the evidence, an open Universe should be preferred." They conclude that "Those cosmologists who take it for granted that we live in a high-density Universe and there seem to be many may turn out to be profoundly mistaken." ${ }^{41}$

Consider also this argument against critical density symmetry. If ours is a critical density closed universe, it must be no older than eight billion years, some astrophysicists indicate. Yet, many galactic structures in the visible universe are older than an eight billion-year-old universe itself! Thus, if criticaldensity cosmologists are right, they must be wrong! Only an open universe allows enough time to account for the age of all its structures. ${ }^{42}$

Trusting experience, the most reasonable thing to believe, though not absolutely certain, is that we live in an open universe. For the net energy of the 
universe to equal zero, all forms of energy, not just a few, must cancel out, including the cosmic contests between matter and antimatter and between gravity and the residual kinetic energy of the Big Bang. They do not balance out. The universe is really something after all! Perhaps it also has a real cause, more than zero existence, and a real purpose!

\section{B. Quantum Natural Laws Operating in Nothingness}

Without realizing or admitting it, Big Accident Cosmologists actually presuppose the existence of something when they imagine the creation of the universe out of and by absolutely nothing. They presuppose the existence of definite laws of nature, specifically those of quantum mechanics, operating on absolutely nothing in a state of absolute nothingness. Our Big Bang and the resulting universe were created, they claim, by quantum fluctuations within nothingness in accord with the laws of quantum physics. According to Stephen Hawking, "It is possible in the quantum theory for the ordinary laws of science to hold everywhere, including at the beginning of time. ${ }^{.43}$ Quentin Smith concedes that the Godless quantum cosmology to which he subscribes,

represents the universe as beginning about 15 billion years ago in accordance with a physical law. The universe is described as beginning from nothing in accordance with some law. Here 'nothing' does not mean the quantum-mechanical vacuum (which it often means in quantum cosmologies, such as Tryon's), but literally nothing, i.e., the absence of all concrete objects (mass, energy, spacetime) ${ }^{44}$

Nothing existed. Yet quantum laws were there. So were quantum fluctuations allowed by these laws. Nothing to fluctuate existed, yet nothingness lawfully fluctuated! Quantum laws existed and produce spontaneous fluctuations within absolute nothingness, says Big Accident cosmology; and this supposedly accounts for the origin of our universe $!^{45}$ But none of this makes good sense, for something (laws and fluctuations) cannot exist in or as absolutely nothing; and no physical laws exist when no physical entities exist. The position is logically incoherent, and it confuses formal with efficient causes. It confuses laws with efficacious energy.

\section{i. The Incoherence of Something in Nothing}

Big Accident Quantum Cosmology cannot be formulated coherently. The difficulties are partly with the fluctuations, partly with the laws. Supposedly, fluctuations in nothingness make bubbles of nothingness that inflate into entire universes of nothingness, one of which is ours. But how can absolutely nothing fluctuate? What could be the difference in meaning or reference between 
"Nothing fluctuated" and "There were no fluctuations"? How could nothing fluctuate at all? How could it fluctuate lawfully? How could "Nothing fluctuated" explain the origin of the universe, especially when the fluctuations themselves were nothing?

Recall that less extreme inflationary theorists derive our universe from the pre-existing "empty space" of Superspacetime, and that this "empty space" has its own physical density and mass/energy. Particles and antiparticles are spontaneously generated by unpredictable fluctuations in a primitive actualized energy field, and an occasional particle that escapes annihilation supposedly inflates into a full-fledged universe. Although primeval inflation within transcendent Superspacetime is very far removed from experience, the quantum-fizzy nature of empty spacetime within our system of nature is well established; and it is not pure nothingness.

As for quantum laws in absolute non-being, Heinz Pagels acknowledged the incoherence of the idea of "laws in nothingness." He wrote,

The nothingness "before" the creation of the universe is the most complete void that we can imagine-no space, time or matter existed. It is a world without place, without duration or eternity, without number-it is what the mathematicians call "the empty set." Yet this unthinkable void converts itself into the plenum of existence-a necessary consequence of physical laws. Where are these laws written into that void? What "tells" the void that it is pregnant with a possible universe? It would seem that even the void is subject to law, a logic that exists prior to space and time. ${ }^{46}$

If laws are there in nothingness, it is not pure nothingness!

\section{ii. Laws Are Only Formal Causes}

Most seriously, in contemporary natural science, physical laws merely describe the statistically average habituated behaviors of actual physical entities, and they change if and when these habits change. Laws are merely formal causes, not efficient causes imposing external limits on what natural entities can do. No abstract laws can exist when nothing else exists for them to describe. Where nothing exists in spacetime, there are no physical laws. No formal causes can operate when nothing has no form, where no habituated energy-laden actualities exist. The actualized energy field of "empty space" has a habitual case of jitters, and so do all the particles that emerge lawfully from it; but it is not sheer nothingness. Quantum laws are finitely probabilistic and predictive, but no finite predictive probabilities exist to be calculate in a state of absolute nothingness. Either primitive physical actualities with habits existed within Big Accident's alleged nothingness, or no quantum laws were there to permit or describe accidental probabilistic world-creating quantum fluctuations of energy-laden 
realities. If quantum laws and primitive physical actualities existed in primeval nothingness, it was not absolute nothingness.

Additional important questions remain unanswered. Do the adduced preworld quantum laws and primitive physical actualities come from somewhere and thus depend on something else for their existence, or are they self-existent, eternal, and necessary beings? If so, by virtue of what do they have this metaphysical status? Why do the laws of quantum physics obtain in nothingness when so many other physical laws-like those of Newtonian mechanics, or of relativity physics alone-are logically possible? Who or what selects the relevant laws? How meaningful is the presumption that totally disembodied laws exist anywhere, much less in absolute nothingness? Big Accident Cosmology has no good answers. It is too much ado about nothingness!

\section{Total Abandonment of Causation}

Some cosmologists admit it, some do not; but the spontaneous creation of our universe occurred only because necessary causal conditions for its creation were met. In that sense, our universe definitely had a cause. Most Quantum Cosmologists are convinced that these necessary conditions include transcendent Mother Spacetime, the physical vacuum, the primordial fizz, an actualized primordial field of energy, and the laws of quantum mechanics.

Extreme Quantum Accidentalists repudiate all of this; they conjecture that our universe is an absolute accident that just popped into being out of pure nothingness devoid of all causal conditions whatsoever. An absolute accident has no causal conditions at all. Accidentalists deny both necessary and sufficient conditions for the existence of our universe. As Stenger says, "The simplest hypothesis that so far seems to explain the data is that the universe is an accident." ${ }^{47}$ What caused the Big Bang? "Nothing!" answers extreme Quantum Accidentalism.

If the Big Bang that created our world was an absolute accident requiring no causal conditions at all, it was the most stupendous accident that ever happened, the greatest miracle of all time, but without a Divine miracle worker. And to think that some people have trouble with God as a miracle worker! Big Accident Cosmologists insist that their creation-by-nothing scenario is all very plausible; but upon closer examination, we see that this is not so.

\section{i. Necessary but Not Sufficient Causal Conditions}

Paul Davies claimed in 1983 that quantum physics abandons causality completely and "permits events to occur without causes in the quantum world." ${ }^{148} \mathrm{An}$ element of truth is in this, but not the whole truth. Spontaneous fluctuations do occur in the quantum world; sufficient causal conditions for spontaneity do not exist. Nevertheless, quantum fluctuations always presuppose and require neces- 
sary causal conditions. Davies concedes as much: "Recent discoveries in particle physics have suggested mechanisms whereby matter can be created in empty space by the cosmic gravitational field, which only leaves the origin of spacetime itself as a mystery." ${ }^{49}$ So both spacetime and a gravitational (or some kind of) energy field are necessary causal conditions for the creation of matter; but how can gravity exist without physical mass? Davies finally concluded that quantum gravity "would allow spacetime to be created and destroyed spontaneously and uncaused in the same way that particles are created and destroyed spontaneously and uncaused." 50 Still, this presupposes quantum gravity, not pure nothingness; but what would quantum gravity attract (or repel) in absolute nothingness? How could it operate in and on nothing? As Einstein insisted, no gravity exists without mass/energy and spacetime. One thing leads to another!

Currently, gravity is best treated within classical relativity physics, which allows for definiteness and continuity in gravitational effects; but no workable theory of quantum gravity is available. ${ }^{51}$ In addition to quantum laws, quantum gravity has its own necessary conditions like gravitational particles-as yet undiscovered gravitons and gravitinos that presently exist in theory only. It also requires mass and spacetime, for gravity is identical with spatiotemporal mass and curvature, according to relativity theory.

Also, in a quantum universe, gravitational particles should display their own quirky quantum effects-indefiniteness, discreteness, spontaneity, scattering, and so forth; but in well-established domains of particle physics, these quantum effects always presuppose their own conditions. When an adequate theory is developed, quantum gravity cannot and will not exclude necessary causal conditions. It will not abandon causation absolutely.

\section{ii. Freedom Has Necessary Conditions}

To turn to another problem, perhaps experience discloses occasional exceptions to the principle of universal causation-like human freedom; and maybe the origin of the universe falls under one of the exceptions.

Freedom and creativity exemplify self-originated spontaneity. This means that things can and do happen without sufficient causes, but not that anything ever happens without necessary causes. This is clearly at odds with the suppositions of extreme Quantum Accidentalists. Contingent realities-things that might or might not be-never come into being when causal grounds, necessary causal conditions, are completely absent. A very decisive empirical consideration supports this: All experience illustrates it. Experience universally confirms that necessary conditions are required for the existence of every contingent entity that comes into being, everything whose non-existence is logically possible, including creativity and acts of free will.

An experienced-based philosophical perspective can make a place for freedom and spontaneity. Both at macroscopic human and microscopic quantum 
levels, creative freedom is incompatible only with sufficient but not with necessary causal conditions. Quantum theory does not dispense with necessary causal conditions, and neither does human freedom. All experience confirms that contingently existing things always have causes, including quantum effects and free choices. Spontaneous quantum fluctuations cannot and do not occur in the absence of necessary causal conditions like Mother Spacetime, or our spacetime, the physical vacuum, an actualized energy field, and quantum laws. Freely made human choices do not occur in the absence of necessary conditions like desires, interests, and some awareness of open possibilities. Without the presence of something, free choices cannot be made. So freedom is no exception to the rule that everything has a cause. Some causal conditions are necessary for the occurrence of everything that happens. Quantum physics and human freedom presuppose only that things can happen in the absence of totally sufficient causal conditions.

\section{iii. Applying Causation to World-Origins}

"Everything that comes into being has a cause" was often defended by rationalistic philosophers like Descartes and Jonathan Edwards as an intuitively certain a priori or self-evident truth. Quite recently, our knowing this principle a priori, without appeal to experience, was vigorously defended by William L. Craig ${ }^{52}$ and attacked (successfully in my view) by Quentin Smith. ${ }^{53}$ To be sure, I, too, find it intuitively obvious that everything that comes into being has a cause; but I cannot rule out the possibility that this powerful intuition is psychological or empirical, not a priori, that it results from (is caused by) its universal confirmation in experience, the last court of appeal in factual matters.

Quentin Smith presents powerful critiques of the empirical argument for the principle of causation and of its application to the creation of the world out of nothing around fifteen billion years ago. The origin of the universe was caused by absolutely nothing, he thinks, because this causal principle is not selfevident and does not apply to the origin of the universe. "Every thing that comes to be has a cause" is well confirmed by experiences of happenings within the world, he admits; but it has no relevant application to the origin of the world itself. $^{54}$ We have no experience of world-causation as such; all relevant experiences pertain only to causation within the world, Smith insists. His view resembles Kant's contention that we have no experience of causation by things in themselves; all relevant experiences pertain only to causation between appearances. Yet, Kant had to explain the existence of appearances causally.

Actually, we have no direct experiences of world-origins from singularities, from "empty space," from quantum tunneling, from God, or from absolute nothingness. So Smith's argument cuts decisively against his own solution to the problem of world-origins. Admittedly, uncertainty creeps into any theistic or non-theistic account of world-creation by God or by any other transcendent 
entities like antecedent universes or Superspacetime. Smith's a-causal Accidentalism is as fatal to Antecedent Universe and Big Fizz Cosmologies as it is to Theism. Uncertainty pervades all of science and philosophy. We are never absolutely sure of the truth of the premises from which we argue; but some premises are still much more plausible than others. Without reiterating Craig's astute responses to Smith, I will offer two important objections of my own to Smith's atheistic account of world-creation from, by, and for absolutely nothing.

First, even Smith does not really believe it. He definitely presupposes the existence and operation of quantum laws, spontaneous fluctuations, quantum tunneling, and a singularity composed of infinitely compressed physical massall in alleged nothingness. Instead of a disembodied God, Smith appeals at rock bottom to the ultimate reality and causal efficacy of disembodied conceptual constructs-quantum laws, fluctuations, tunneling, and a sizeless, timeless, and imperceptible "physical" singularity. Without them, Smith has no explanation of the origin of the universe from and by nothingness. Even without his singularity, Big Accident Quantum Cosmologists always presuppose the antecedent reality of quantum laws, perturbations, tunneling, or something within absolute non-being; but they pronounce their theory only at the price of incoherence. Their nothingness is not real nothingness; it is really something after all!

Second, recall Smith's argument that our knowledge of causation, drawn from within the world, cannot be applied to the origin of the world because we have never experienced world-origins. But Smith's Accidentalist account of origins is vulnerable to exactly the same objection. In quantum physics, he claims, particles can come into existence spontaneously under conditions of quantum uncertainty, and the law of energy conservation does not apply to these circumstances. Once, such a particle inflated into our universe. But note carefully that Smith's theory of world-origins depends entirely upon an analogy with experienced quantum fields and processes within our world. As he puts it, "There is observational evidence, albeit indirect, that this uncaused emergence of energy or particles (notably virtual particles) frequently occurs." 55 This could be true only within our system of spacetime, if Smith is really serious about appealing to experience. We have no "observational evidence" of world-origins.

Smith's position is implausible for many reasons. No one has ever seen a quantum particle inflate into an entire universe. All our empirical or observational knowledge of quantum effects is based upon experiences of quantum events within our world. If knowledge of universal causation drawn from within the world cannot be applied to the origin of the world, then knowledge of quantum effects drawn from within the world cannot be so applied either. Also, physicists have proved that only a closed universe could arise through quantum tunneling, ${ }^{56}$ the process by which virtual particles become actual; but ours is not a closed universe, as earlier demonstrated. Thus, Smith cannot make his case for thinking that our world is a quantum-induced Big Accident. 
We have no experience of world origins through spontaneous quantum fluctuations in absolute nothingness. All human experiences of quantum phenomena pertain only to minute quantum events within our system of spacetime. If we cannot extrapolate from the universality of causation within the world to world origins, neither can we extrapolate from quantum effects within the world to world origins. All experienced quantum effects, including those in virtually empty space, presuppose not only quantum laws but also our space, not transcendent Superspace, and definitely not pure nothingness. Empirically, the spontaneous origin of actualized particles under conditions of Heisenberg uncertainty occurs only under vacuum conditions within our system of spacetime, never in absolute nothingness; and few if any such particles endure for more than a fraction of a second to violate the Principle of Conservation.

Smith actually concedes that all known quantum tunneling, by which, in theory, particles momentarily break the barrier separating potentiality from actuality, takes place within our system of spacetime. ${ }^{57}$ All known tunneling and quantum effects presuppose necessary causal conditions like our spacetime, vacuum (low energy) conditions, an actualized energy field, and perhaps even observers or prehenders (not necessarily conscious). From nothing, nothing comes, even in quantum physics.

Smith's own account of world origins is incompatible with his argument against "Everything that comes to be has a cause." It presupposes and thus grants that we can extrapolate from what is known within our spacetime to the origin of the whole of it. But if we can apply what we know about quantum laws and effects to the origin of our universe, we can also apply the universally confirmed principle of causation to world origins. That rebuts Smith's No-cause Big Accident theory of the inception of the universe. Unless everything that comes into being, including the universe, has a cause (presupposes necessary conditions), no Quantum Cosmology ever gets off the ground. Big Accident's misunderstanding of quantum physics can be extended to the whole of nature only if explanatory principles drawn from the parts can be so extended; but that lets causation back in under the wire. Any account of the origin of our universe must draw upon analogies with what happens within our universe, for that is all that we know.

Stephen Hawking maintains that "It is possible in the quantum theory for the ordinary laws of science to hold everywhere, including at the beginning of time." ${ }^{8}$ If, as Smith and Hawking suggest, the laws of quantum physics do not break down at the origin of the universe as a whole, then neither does the law of universal causation, nor the law of increasing entropy. If any explanatory principles can be extrapolated from parts to the whole, surely the universality of causation is one of them. Contra Smith, a quantum universe presupposes necessary causal conditions. But, deep down, Smith really believes that anyway.

The ad hominem retort that "You do it too!" will not satisfy extreme skeptics. Still, anyone, whether an atheist or a theist, who attempts to answer the 
question, "What caused the Big Bang?" must employ concepts and principles of explanation drawn from within the universe and extend them to the origin of the whole. We could just abstain or quit trying to find a cause for the Big Bang, but the inclination to try is almost irresistible. Anyone who decrees that it simply cannot be done merely blocks the path of inquiry.

The causal principle that "Everything that comes to be has a cause," at least as necessary conditions, must be distinguished from what Victor J. Stenger calls the principle of "causal precedence," that "Cause always precedes effect." Stenger argues that at the elementary level of quantum interactions, "Cause and effect are not always distinguishable" because time is reversible. The irreversibility of time's arrow, he maintains, is an emergent property that exists only at the macroscopic level of everyday experience and common sense. ${ }^{59}$ The solution to the two slit experiment problem, he holds, is that a particle goes "through one slit to the detector, then back in time to the source through the second slit and finally forward in time once more through either slit to the detector." ${ }^{10} \mathrm{He}$ concedes that he is "in a minority" on the issue of the reversibility of time! " $^{61}$

That everything which comes into being has a cause would still be true even if we cannot always tell the difference between cause and effect, or if cause, effect, and time are reversed on the quantum level. Stenger, another Big Accident Cosmologist, also affirms that "everything can have come from nothing" in the beginning ${ }^{62}$ through quantum fluctuations in the spacetime vacuum. We now know that this actually involves the antecedent reality of necessary causal conditions like Mother Spacetime, the physical vacuum, actualized energy, habituated actualities, quantum laws, and quantum effects. In their absence, spontaneous fluctuations cannot and do not occur. All contingencies have causes.

\section{No Contingency Without Causation}

By definition it is true that if something exists contingently, it is causally derived from or dependent on something other than itself; but a mere definition cannot settle the substantive question of the causal dependence of the universe on God. Can a wedge be driven between the various elements that conventionally define the notion of contingent existence? Could an existing entity that endures for only a finite span of time have no cause at all even though it is possible for it not to exist? The most extreme Big Accident Cosmologists think so. They contend that the universe popped into being within the finite past as an absolute accident, requiring neither necessary nor sufficient causal conditions. If true, the universe could be contingent (having possible non-existence and finite duration), yet-in another sense-neither contingent (causally dependent) nor necessary (impossible non-existence, self-sufficient, everlasting, uncreated, and indestructible reality). 
Big Accident Cosmologists appeal to quantum theory, which really does not support their position because quantum mechanics renounces only sufficient but not necessary causal conditions. Every quantum fluctuation has necessary prerequisites like the laws of quantum physics, spacetime or Superspacetime, the minimal pure energy of a physical vacuum, and the presence of an actualized energy field. They aspire to do so, but Big Accident Cosmologists cannot avoid these necessary conditions for quantum fluctuations, primordial or not; but primordially where and how did these conditions originate? They are not metaphysically necessary beings because as wholes they are composed entirely of contingent, dependent parts. This point is well developed in Chapter Twelve, but let us now note that Big Accident Cosmology falls prey to the first two premises of the Cosmological Argument From Contingency there presented. All wholes composed of contingent beings are themselves contingent; and no necessary entities exist within our system of nature.

To summarize, extreme Big Accident Cosmology takes quantum fluctuations to their ultimate extreme and posits their reality where nothing exists to fluctuate. Our universe was created out of absolutely nothing, was caused by absolutely nothing, is absolutely nothing, and exists for absolutely no purpose. It is the Ultimate Accident. Extreme Accidentalism assumes that our universe is composed of zero energy, and that since the universe is nothing, nothing is required to create it. But a zero energy universe is very doubtful-especially ours. The kinetic energy of the primordial explosion that initiated our universe is not perfectly counterbalanced by gravity, and matter clearly prevails over antimatter in the only universe that we really know to exist. A zero-energy universe exists only in theory, but experience clearly shows that our universe is really something after all!

Extreme Accidentalists always presuppose something as a necessary ground for the universe, even if nothing more than the laws of quantum physics and spontaneous fluctuations. They fail to distinguish adequately between necessary and sufficient causal conditions. If the Big Bang lacked a sufficient cause, it does not follow that it had no necessary cause. All experience, including quantum physics, supports the causal principle that some causal conditions are necessary for everything that happens or comes to be, including the Big Bang. Scientific Cosmological Agnosticism correctly indicates that empirical science cannot identify the transcendent necessary cause of the Big Bang.

Big Accident Cosmologists have wild imaginations, but their theory is logically incoherent and cannot be squared with the facts. Like all other atheistic Quantum Cosmologists, they are driven to preposterous extremes in order to avoid God. A contingent universe cannot exist in the absence of everything; but what is the something upon which it depends for its being? What caused the Big Bang? No atheistic cosmologies examined thus far, quantum or not, give an adequate answer; but a plausible answer is forthcoming! 